\title{
La poétique de la contradiction chez Nelly Arcan
}

\section{Laurence GAUVREAU, Université de Montréal (BACC)}

L'autofiction chez Nelly Arcan, en plus de mettre en scène l'union atypique, bien que déjà vue, de l'écriture et de la prostitution, donne l'impression d'être tout entier un rassemblement de pensées opposées, incompatibles, en désordre. S'opposer à soi-même peut montrer l'existence d'un esprit critique, en plus de la capacité à pousser celui-ci au-delà du simple débat entre amis. La critique négative et la négativité de la critique pénètrent profondément le processus d'écriture dans les autofictions d'Arcan, Putain et Folle, et cette dernière possède définitivement un esprit critique. Par contre, celui-ci ne semble pas la source des oppositions qui caractérisent sa plume. La poétique de l'auteure semble en effet être fondée sur l'utilisation constante d'une série de contradictions. C'est dans la tension de l'exploration de ces contradictions, de la force de cette dialectique négative, qu'Arcan tente de faire éclater le sens des préjugés, de ce qui a été jugé d'avance sans aucun esprit critique, afin d'atteindre une conception et une compréhension qui seraient plus authentiques.

Les conclusions de cet article s'appuient sur la théorie de l'autofiction. À la suite de la lecture de plusieurs œuvres théoriques sur ce genre ; ayant comme auteurs Serge Doubrovsky, Philippe Pouliot, Sébastien Hubier, Philippe Vilain, Madelaine Ouellette-Michalska, Jean-Louis Jeannelle ou Catherine Viollet; une définition de l'autofiction peut être établie, sur laquelle s'appuiera cet article. D'abord, l'autofiction est une œuvre dans laquelle il doit y avoir un rapport entre la vie de l'auteur et le texte, c'est-à-dire une homonymie entre narrateur, auteur et personnage, de sorte que le héros et/ou le narrateur doit porter le nom de l'auteur. Cela permet, entre autres, de différencier l'autofiction du roman autobiographique et rend l'identification personnage/auteur/narrateur évidente. Même si la biographie de l'auteur est connue du grand public, on ne peut prétendre que tout lecteur verra ces références dans le texte. Ensuite, l'autofiction est un croisement, un genre hybride entre le roman et l'autobiographie, c'est-à-dire un texte dans lequel, comme le dit Sébastien Hubier (109-129), se retrouvent trop d'éléments associés à la vie de l'auteur pour qu'il s'agisse d'un roman et trop de fiction pour qu'il s'agisse d'une autobiographie. Peu importe le genre dicté par le paratexte; l'autofiction se définit justement par son ambiguïté et mélange générique. Puis, comme l'affirme Philippe Vilain (52- 
57), la part de fiction de l'autofiction doit être intentionnelle de la part de l'auteur. Les textes autobiographiques dans lesquels des défaillances de la mémoire créent une part de «fiction » ne sont pas des autofictions. Enfin, l'idée que l'autofiction se caractérise par une auto-analyse de l'auteur est rejetée. Comme le dit Philippe Pouliot (11-42), on ne peut ignorer qu'il s'agit d'un élément récurrent qu'il est important de théoriser sans pour autant qu'il s'agisse d'une caractéristique de toutes les autofictions. Il est évident qu'une autofiction est un texte riche pour un psychanalyste de la littérature : les choix de l'auteur en disent beaucoup sur sa psyché. Par contre, l'écrivain peut écrire une autofiction sans consciemment vouloir s'analyser, bien que son texte permette à un autre de le faire. Ainsi, l'autofiction est un texte ayant toujours un rapport avec la vie de l'auteur, c'est-à-dire un croisement entre récit d'expériences vécues (autobiographie) et fiction intentionnelle de l'auteur (roman) et dont le héros et/ou narrateur partage l'identité de l'auteur en portant son nom.

Ainsi, cet article explique d'abord la source des contradictions qui abondent dans les autofictions de Nelly Arcan. Ensuite, il sera montré les échos des pensées opposées de la narratrice sur l'échange de cette dernière avec elle-même et sur l'échange avec ses proches. Puis, la perception changeante que la narratrice a de sa quête d'authenticité à travers l'écriture sera exposée, expliquant également certaines des paroles antithétiques de celle-ci.

\section{Deux discours}

La poétique des deux autofictions de Nelly Arcan peut être considérée comme contradictoire puisque la narratrice de ces œuvres est prise entre deux discours. Le premier est lié à l'être fondamental qu'elle est, en dehors du jeu des faux-semblants sociaux. Cette dernière semble en effet rechercher une certaine authenticité dans ses récits. Elle affirme même qu' «écrire exigeait un laisser-aller de sa personne » (Folle, 165) et la préface de son premier roman, Putain, laisse entendre qu'elle «écrira jusqu'à grandir enfin» (18). Se laisser aller, grandir : voilà des actions qui semblent demander ou créer un contact avec soi-même comme être vrai et authentique.

Le deuxième discours a été engendré par la société dans laquelle la narratrice évolue. Il s'agit d'un discours par lequel elle se construit afin de correspondre à ce que cette société lui dicte d'être pour être acceptée et plaire : une femme soumise à l'homme, réduite à son sexe, silencieuse et se conformant aux critères de beauté d'une époque où l'artifice, le maquillage et la 
chirurgie esthétique sont à portée de main. En fait, la narratrice ne fait pas que constater ces règles du jeu de (la) société, mais les intègre à sa vie. Arcan situe donc l'existence de la narratrice au milieu d'un système social auquel cette dernière tente de correspondre et d'adhérer. En essayant de reproduire le modèle de la femme engendré par ce système, la narratrice est complice de sa propre aliénation, créant de toute pièce certaines facettes d'elle-même. Il devient ainsi parfois ardu, voire impossible, pour la narratrice de ne pas constamment jouer un rôle, de ne pas être constamment consciente de ce qu'elle renvoie comme image. En effet, elle a intégré à sa vie les règles de la société, mais jusqu'à ce que celles-ci la dépassent, jusqu'à ce que le cadre dans lequel elle vit devienne parfois plus fort que sa volonté. La narratrice participe ainsi à ce qu'elle tente de critiquer, c'est-à-dire l'image de la femme objet tentant de ressembler à ce que Claudia Labrosse nomme comme le «mythe de la beauté »(25). En bref, Labrosse définit ce mythe comme étant l'existence d'une beauté universellement reconnue. De plus, la narratrice admet même que «le besoin de plaire l'emporte toujours lorsqu'[elle] écri[t]» (Putain, 17), action qui devrait normalement engendrer un certain abandon ou un laisser-aller, comme elle le dit elle-même. C'est ainsi qu'Arcan montre que chaque action, chaque geste, chaque choix de ses personnages féminins sont guidés par cette force sociale qui les oblige à se plier à ses lois. Force qui peut finir par apparaitre comme naturelle à force de se performer constamment, même lorsque les femmes sont seules face à elles-mêmes.

L'écriture d'Arcan laisse ainsi sentir que le cadre dans lequel la narratrice vit, la société, empêche cette authenticité ou, du moins, la rend difficile d'accès. Cette quête d'authenticité est un projet inachevable dans les œuvres d'Arcan. De l'opposition entre les illusions sociales de la féminité et le désir des femmes d'être authentiques résulte une lente destruction du sujet féminin. Déchirés par cette opposition, les personnages féminins sont inévitablement condamnés à l'anéantissement dans les autofictions d'Arcan. Il existe ainsi une tension chez la narratrice entre l'image qu'elle se fait d'elle-même et qu'elle désire projeter (le deuxième discours), et les réponses la concernant qui pourraient remettre en question ce discours, c'est-à-dire sa recherche d'authenticité (le premier discours). Le processus de création littéraire est donc pris, chez Arcan, entre la renonciation face à l'aliénation sociale et la possibilité de donner forme à une expérience authentique de l'être humain. D'ailleurs, l'échange mis en scène dans les autofictions d'Arcan est fortement influencé par ce double discours de la narratrice. 


\section{L'échange contradictoire}

L'échange est ici plus que le fait de donner et de recevoir en contrepartie. Il s'agit plutôt d'un échange d'ordre communicationnel. Cet échange communicationnel est l'action intéressée d'adresser (de «donner ») à un destinataire un message qu'il peut décoder, qui suppose une réception et dont la réciprocité (recevoir en contrepartie) est théorique dans l'échange, mais non essentiel dans le processus de communication. La réciprocité de l'échange communicationnel est donc théorique et supposée, mais pas nécessairement présente. Dans les autofictions d'Arcan, la narratrice s'adresse effectivement d'abord à des lecteurs, mais aussi à son ex-amant (au «tu »), à ses anciens clients et indirectement à ses parents (en leur faisant des reproches et en parlant d'eux, par exemple). Elle le fait à l'aide d'un message qu'ils peuvent théoriquement décoder, c'est-à-dire l'écriture en langue française. Ce message engendre une réception puisque plusieurs lecteurs ont acheté, lu et même commenté les œuvres autofictionnels de Nelly Arcan. Par contre, cette réception est supposée chez les proches de la narratrice. Écrivant une autofiction, cette dernière met en scène ses proches, qui s'y sentent forcément interpelés et possiblement curieux de la lire. Le retour de cet échange semble par contre plus complexe. Il est parfois présent, parfois absent, parfois camouflé ou esquiver, nous le verrons.

L'échange de la narratrice qu'Arcan met en scène, dans les deux autofictions que sont Putain et Folle, est ainsi contradictoire puisqu'également pris entre recherche d'authenticité et construction de soi amenée par les exigences sociales. La volonté de la narratrice de se parler ou de communiquer avec ses proches est mêlée à un désir, conscient ou pas, d'esquiver cet engagement et surtout le retour de cet échange, puisque ceux-ci pourraient remettre en cause la construction d'elle-même que la narratrice a bâtie. La narratrice semble avoir peur de voir audelà du discours sur elle-même qu'elle s'est fabriquée. Peur également causée par une sensation plutôt négative lorsqu'elle tente l'expérience, une «écriture nocive » (Folle, 142), comme le remarque l'ex-amant de la narratrice.

De ce fait, la narratrice se questionne, se regarde dans le miroir et tente de se livrer sans censure à travers l'écriture. Il s'agit là de sa quête d'authenticité lorsqu'elle échange avec ellemême. Par contre, l'appréhension et la crainte de se voir et de se répondre réellement, sans fiction ou artifice, créent cette tension perceptible à travers sa volonté de camoufler cette adresse à elle-même. Par exemple, la narratrice utilise la figure du psychanalyste, dans Putain, afin de se poser des questions qu'elle n'oserait peut-être pas se lancer directement. En effet, le 
psychanalyste reste «invariablement muet» (Bordeleau, 22) dans Putain et la narratrice s'en moque même à plusieurs reprises. Pourtant, cette figure permet à cette dernière de se questionner. La narratrice s'oblige à faire un retour sur elle-même, mais l'adresse au psychanalyste laisse croire qu'il s'agit d'une démarche imposée par celui-ci. Ainsi, se retrouvent dans le premier récit d'Arcan, Putain, des formules comme « je dois maintenant me rappeler quand et comment tout ça a commencé » (55), «je dois savoir pourquoi il en est ainsi » (79) ou «je dois parler de tout ce que je vous jette à la figure » (100). La narratrice joue donc l'analysant et l'analysée, exigeant d'elle-même des réponses et des réflexions, mais tente de le camoufler en donnant l'impression que ces exigences viennent d'un autre. Comme la narratrice ne rapporte jamais les paroles du psychanalyste ou le fait parler au conditionnel, le présumé discours de celui-ci semble en fait cacher le sien. De plus, cette tension n'est pas seulement visible dans l'engagement de l'échange avec elle-même, mais aussi lorsque la narratrice tente d'esquiver ou de diminuer l'impact du retour de cet échange, c'est-à-dire les réponses qu'elle se donne. Ainsi, elle peut par exemple affirmer que «les miroirs ne [lui] renvoient plus qu'une doublure » (Putain, 25), ce qui laisse croire qu'elle ne se permet de voir dans le miroir que la partie d'ellemême qui ressemble aux autres femmes, tentant de correspondre aux mêmes critères de beauté qu'elle. Se voir comme une doublure suggère une absence d'unicité chez la narratrice, comme si ce qu'elle veut ou se permet de voir d'elle-même ne sont que les aspects qui ressemblent aux autres et qui entrent dans la norme établie. Ainsi, en plus de ne voir d'elle-même que ce qui correspond aux critères de beauté de son époque, elle éviterait aussi peut-être de voir au-delà de son apparence. En ce sens, elle affirme que si elle creuse en elle, «ce qu'on y trouverait serait toujours à enlever, couche par couche » (Putain, 41-42). Ce qui la constitue réellement sous son apparence semble donc vouloir être évité, sans intérêt pour elle. Cela montre un désir ou une pulsion chez elle de ne pas regarder plus loin que son enveloppe, c'est-à-dire ce qu'elle se permet de voir d'elle-même dans le miroir. Une autre stratégie est de ne s'y voir que par parties : «je ne veux me regarder que par petits coups d'œil, que du bout des yeux, je n'ai pas envie de me voir à ce point-là » (Putain, 128). Bref, la tension contradictoire se met ici en scène car, malgré la volonté de la narratrice de vouloir échanger authentiquement avec elle-même, cette dernière tente consciemment ou pas d'esquiver cet échange.

L'échange de la narratrice avec ses proches reflète également cette tension contradictoire. D'abord, comme le démontre Annie Richard tout au long de son livre L'autofiction et les 
femmes: un chemin vers l'altruisme?, le statut autofictionnel d'une œuvre interpelle inévitablement les proches de l'auteur puisqu'une partie du récit est annoncée comme vrai et inspirée de la vie de l'écrivain. Ensuite, la narratrice exprime elle-même un désir de communication avec ses proches. En effet, en plus de critiquer ses parents tout au long de Putain et Folle, elle affirme qu' " écrire voulait dire ouvrir la faille [...] il fallait pour y parvenir choisir ses proches et surtout il fallait les avoir follement aimés, il fallait les pousser au pire d'euxmêmes et vouloir leur rappeler qui ils sont. » (Folle, 168) Ces deux faits montrent que la narratrice désire engendrer une communication authentique avec ses proches : elle veut leur parler sans détour, leur faire voir leurs failles et ce qu'elle pense d'eux sans retenue. Par contre, le fait de leur parler peut générer un retour, créant un échange, qui pourrait remettre en question l'image qu'elle se construit d'elle-même. La narratrice met donc encore en place des stratégies pour éviter ce retour, c'est-à-dire ici l'évitement d'un contact direct avec ses proches. Par exemple, Arcan met en scène une narratrice qui parle de ses parents sans jamais leur parler. Par exemple, elle leur pose constamment des questions indirectes : «à quoi pense-t-elle [sa mère] lorsqu'elle se voit si jeune et si belle » (Putain, 107), « qu'aurait-il [son père] fait s'il l'avait su » (Putain, 114). De plus, ses proches ne s'expriment que lorsque la narratrice rapporte leurs paroles passées. Il y a donc parfois apparence de dialogue au présent entre eux, mais en réalité la narratrice ne fait que les citer. Elle leur répond en fait à retardement, sans que ses proches aient une voix actuelle et au présent. Les seules manifestations non révolues de ces derniers se mettent en scène au conditionnel présent ou passé. Ainsi, la narratrice va même jusqu'à fabuler leurs paroles pour, encore une fois, éviter de leur donner une voix au présent : « tu pourrais dire qu'au fond, ce n'est pas si grave [...]. Tu pourrais dire que le geste de cracher n'est pas si différent de celui de marquer l'autre avec ses dents [...]. Tu pourrais dire que mon passé de pute m'a fait voir et entendre le pire [...]» (Folle, 29-30). Faire parler ses proches au passé ainsi qu'assumer ce qu'ils diraient ou aurait dit montrent ainsi des stratégies d'évitement d'un discours, ici la voix de ses proches, qui pourrait contredire le discours par lequel elle se construit. Elle refuse d'entendre ce que ses proches ont à dire d'elle afin de garder le contrôle sur ce qu'elle croit projeter. Le discours au présent de ses proches fait ainsi partie du premier discours, celui qui a le pouvoir de remettre en cause le deuxième. La volonté d'échanger avec ses proches, mêlée simultanément à l'évitement de cette action, montre encore la tendance contradictoire qui qualifie l'échange chez la narratrice de Putain et Folle. 


\section{La quête de la narratrice bouleversée par l'écriture}

C'est à travers l'écriture autofictionnelle que la narratrice constate que la construction qu'elle s'est faite d'elle-même rend difficile d'accès ce qu'elle est vraiment, en dehors des règles qu'impose la société pour être acceptée, plaire et considérée comme belle. De ce fait, son être fondamental n'existerait pas totalement : « et qu'y aurait-il en dessous pensez-vous, sous la surface de ce qui est à enlever, [...] ce qu'on y trouverait serait toujours à enlever, couche par couche [...]»(Putain, 41-42). La narratrice se considère ainsi que comme une série d'apparences. Pourtant, elle est bien autre chose que cela; elle l'entrevoit même à travers son processus d'écriture lorsqu'elle affirme que «[...] il me semblait qu'en écrivant on ne libérait rien du tout, que plutôt on s'aliénait $[\ldots] »($ Folle, 172). Si l'écriture la rend étrangère à ellemême, c'est donc que celle-ci lui fait voir une autre partie d'elle-même, méconnue jusqu'ici et donc non circonscrite par les idéaux sociaux. De plus, à la suite de ses récits, elle conclue même qu' « écrire ne sert à rien, qu'à s'épuiser sur de la roche ; écrire c'est perdre des morceaux [...] » (Folle, 205). L'écriture lui a donc fait voir des strates d'elle-même qu'elle ne voulait finalement pas rencontrer et qui auraient peut-être mieux fait, selon elle, de rester cachées et incomprises. L'absence d'un être fondamental chez la narratrice semble donc être une volonté plutôt qu'un constat. Elle recherchait l'authenticité, mais conclue, consciemment ou pas, qu'elle préfère peutêtre n'être qu'une construction. En effet, tout ce qui pourrait contredire l'image d'elle-même qu'elle s'est créée, c'est-à-dire le retour de l'échange avec elle-même ou le regard et l'opinion de ses proches, semble lui déplaire et la déstabiliser, d'où la tendance de la narratrice à fuir, camoufler ou esquiver tout ce qui contredirait cette construction, ce qu'elle semblait pourtant rechercher au départ. La peur de se voir réellement semble donc souvent l'emporter sur sa recherche d'authenticité, elle-même entravée par les exigences de la société dans laquelle elle évolue. La narratrice semble l'avoir compris par le biais de l'écriture. Il y a donc chez elle une tension entre la volonté de montrer le vrai et une forte attirance pour le faux, qui camouflerait cette vérité parfois difficile à voir. Ne serait-ce d'ailleurs pas là l'essence de l'autofiction, c'està-dire la réunion de l'autobiographie et du fantasme, une version améliorée, idéale ou transformée de notre vie? 


\section{La limite entre authenticité et construction de soi}

Arcan semble créer une sorte de mise en abyme dans ses autofictions : une autofiction dans une autre. Le personnage narrateur qu'elle met en scène éprouve certainement parfois des difficultés à tracer une ligne entre les traits de sa personne qui lui ont été imposés ou transmis par la société dans laquelle elle évolue et ceux qui la constituent en dehors de celle-ci, bien qu'elle est parfois consciente de la différence entre les deux. En effet, comme énoncé précédemment, si cette construction sociale apparait parfois comme naturelle, il devient ainsi difficile de départager celle-ci et l'authenticité recherchée. D'où le projet ardu, voire inachevable, de sa quête d'authenticité. Où se trouve la limite entre ce que je suis réellement et ce que je performe être au quotidien ? La force extérieure qu'est la société peut être intériorisée à un tel point que les femmes la ressentent comme étant naturelle, comme émanant naturellement de leur être le plus profond. Ainsi, certains traits de la construction que la narratrice se fait d'elle-même ne peuventils pas finir par paraitre, à ses yeux et aux yeux de tous, comme une essence ? Oui, la société lui a imposé une vision de la femme et de la beauté, mais cela s'est produit dès l'enfance et surtout à l'adolescence, berceau de la formation de notre personnalité. Est-elle ou a-t-elle déjà été quelque chose d'autre que cette construction? La narratrice ne se prononce pas directement sur cette question, mais comme montré précédemment, la façon dont elle parle d'elle semble remettre en question l'existence d'un être fondamental, sans pour autant en énoncer la certitude puisqu'elle semble voir dans le miroir certaines parties d'elle-même sur lesquelles elle n'a aucun contrôle. La narratrice expérimente ainsi, à travers l'écriture de soi, un brouillage générique à l'intérieur de sa propre vie, de ses propres choix, de sa propre personnalité. Un brouillage entre essence et construction. Tout comme le lecteur des autofictions de Putain et Folle expérimente un brouillage générique entre biographie et fiction, duquel il ne saura jamais avec certitude quelle part est véridique ou inventée. L'autofiction mêle ainsi expériences vécues et fiction, tout comme la narratrice des autofictions d'Arcan mêle ce qu'elle est et ce qu'elle voudrait ou performe être.

\section{Les contradictions nouées}

Il est ainsi facile de voir pour quelles raisons la narratrice semble confuse en ce qui concerne l'établissement de son individualité et de son identité. Elle croyait peut-être définir cette identité par l'écriture : en jouant au psychanalyste avec elle-même, en disant à ses proches ce qu'elle n'a jamais osé leur dire, en se fixant comme but de «grandir enfin ». Par contre, la 
rédaction semble l'avoir rendue davantage confuse, consciente de ce brouillage générique qu'est sa personne : «ce dont je devais venir à bout n'a fait que prendre plus de force à mesure que j'écrivais, ce qui devait se dénouer s'est resserré toujours plus jusqu'à ce que le nœud prenne toute la place [...] » (Putain, 17). Son être est ici comparé à un nœud : entrelacement de deux ou plusieurs extrémités dans le but de les réunir. Sa propre personne semble ainsi réunir « deux extrémités » (être authentique et construction sociale), tout en rassemblant des facettes d'elle qui se brouillent, formant un tout dont les éléments ne sont plus dissociables, à la manière d'un nœud. La narratrice est donc non seulement prise entre deux discours la concernant, mais ne fait parfois plus la distinction entre les deux. Cela engendre à la fois la possibilité d'un échange avec elle-même et une tension entre deux extrêmes qui sont difficiles à départager. Plutôt que la présence d'un esprit critique, voilà la source des incessantes contradictions qui représentent l'essence, cette fois non mêlée aux dictats sociaux, de l'écriture unique, noire et touchante de Nelly Arcan.

\section{Bibliographie}

Arcan, Nelly. Folle. Paris : Seuil, 2004.

---. Putain. Paris : Seuil, 2001.

Bordeleau, Francine. «La mise en scène de l'autofiction ». Spirale 182 (2002). 22.

Hubier, Sébastien. «Aux lisières de la vie et de la fiction ». Littératures intimes - les expressions du moi, de l'autobiographie à l'autofiction. Paris : Armand Colin, 2003. 109-129.

Labrosse, Claudia. «L'impératif de beauté du corps féminin : la minceur, l'obésité et la sexualité dans les romans de Lise Tremblay et de Nelly Arcan ». Recherches féministes 23 (2010). 25-43.

Pouliot, P. «Chapitre 1 : autofiction et fiction de soi : considérations théoriques », « Fiction de soi et autoréflexivité romanesque dans la littérature québécoise contemporaine (19952000) ». Mémoire de maîtrise, U de Montréal, 2002. 11-42.

Richard, Annie. L'autofiction et les femmes : un chemin vers l'altruisme? Paris : Harmattan, Coll. Espaces Littéraires, 2013.

Vilain, Philippe. «Définir malgré tout ». L'autofiction en théorie. Chatou : Transparence, Chatou, 2009. 52-57. 